Pure and Applied Mathematics Quarterly

Volume 7, Number 4

(Special Issue:

In memory of Eckart Viehweg)

$1785-1809,2011$

\title{
Cuspidal Curves and Vector Bundles on an Algebraic Surface, $\mathbf{I}^{*}$
}

\author{
Sheng-Li Tan
}

\section{Dedicate to the memory of Eckart Viehweg}

\begin{abstract}
We use vector bundle method to study cuspidal curves $C$ on an algebraic surface $X$. We prove that $C$ is the branch curve of a generic triple cover over $X$ iff the cusps on the curve are 3-divisible. We obtain a numerical condition on $C$, by using Miyaoka-Yau's inequality, such that the number of generic triple covers ramified over $C$ is determined. We get also an optimal lower bound on the number of cusps on such a branch curve by using Bogomolov's inequality. As an application, for each rank two vector bundle $E$ on $X$, we associated it with an irreducible cuspidal curve $C$ whose cusps are 3-divisible and $E$ is determined uniquely by $C$ and the 3-divisibility of the cusps.
\end{abstract}

Keywords: cuspidal curve, vector bundle, algebraic surface, triple cover, 3-divisible set.

\section{INTRODUCTION}

In classical algebraic geometry, every projective surface $Y \subset \mathbb{P}^{N}$ is expressed as a multiple plane $\pi: Y \rightarrow \mathbb{P}^{2}$ via a generic linear projection from the projective space to the projective plane, the branch curve $C$ is an irreducible cuspidal plane curve of even degree $2 d$, namely, $C$ admits only nodes and ordinary cusps as its

Received: Oct. 16, 2010; Revised: Feb. 20, 2011.

*This work is supported by the NSF of China (No: 10731030), Science Foundations of the Education Ministry of China, STCSM Foundation of Shanghai. 
singularities, and the finite cover is a generic cover. O. Chisini [6] summarized the following 4 fundamental problems in this classical study.

(1) Characterize those plane curves that are branch curves of some multiple planes, and for each of them construct effectively the corresponding finite covers.

(2) Determine birationally all multiple planes with the same branch curve.

(3) Construct some examples of notable surfaces by using the correspondence between the branch curves and the multiple planes.

(4) Consider a multiple plane by means of its branch curve, determine its characters.

Furthermore, Chisini [6] pointed out that the first problem is the most important one. The main purpose of this study is to reduce the classification of algebraic surfaces to that of cuspidal branch curves.

In fact, the second problem is completely solved by Kulikov [11, 12] and Nemirovskij [16]. The surface $Y$ and the covering $\pi: Y \rightarrow \mathbb{P}^{2}$ are determined uniquely by its branch curve $C$ except for the case when $Y \cong \mathbb{P}^{2}$ is the Veronese surface in $\mathbb{P}^{5}$ and $\pi$ is a generic projection (Chisini's Conjecture [5]). In the exceptional case, the branch curve is a sextic with 9 cusps and the degree of $\pi$ is 4 . (In fact, there is one generic triple cover branched along this sextic.)

The first problem was first considered by F. Enriques [7] in 1923, he gave a topological characterization on the branch curves $C$ based on its fundamental group $\pi_{1}\left(\mathbb{P}^{2} \backslash B\right)$. Because we know very little about this fundamental group, algebraic geometers have been looking for a pure algebro-geometric characterization for a long time [13]. O. Zariski [23] found the first such characterization in 1929: a sextic with 6 cusps is a branch curve if and only if the 6 cusps lie on a conic. Furthermore, he developed a general method to compute the fundamental group in the same paper. When $Y$ is a smooth surface in $\mathbb{P}^{3}$ and $\pi$ is the generic projection with branch curve $C$, B. Segre [17] gives a complete answer.

The first non-trivial case of multiple planes is triple planes. The branch curves contain only cusps as their singularities. P. Du Val [3, 4] studied the branch curves of small degrees. In a series of papers, Bronowski [2] studied the lower and upper bounds on the number of the cusps on the branch curve of a triple 
plane. In [20], M. Teicher and the author classified completely all such curves up to degree 12 .

The purpose of this paper is to use rank two vector bundle method to study cuspidal curves on an arbitrary projective smooth surface $X$.

To state our main results, we first set up the following definitions:

Definition 0.1. A generic cover between two smooth algebraic surfaces $X$ and $Y$ is a finite morphism $\pi: Y \rightarrow X$ satisfying the following conditions:

(1) The branch curve $C$ of $\pi$ is an irreducible curve with nodes and ordinary cusps as its singularities;

(2) $\pi^{*}(C)=2 R+D$, where $D$ is reduced and the restriction map $\left.\pi\right|_{R}$ from $R$ to $C$ is the normalization of $C$.

We consider the general case where the branch curve admits at least one singular point. The case with smooth branch curve is easy to deal with.

Let $C$ be an irreducible curve on $X$ with $n \geq 1$ ordinary cusps $\left\{p_{1}, \cdots, p_{n}\right\}$. Suppose $C \equiv 2 \eta$ is an even divisor, $\varphi_{\eta}: \Sigma_{\eta} \rightarrow X$ is the double cover ramified over $C$ determined by $\eta$. Let $S_{\eta} \rightarrow \Sigma_{\eta}$ be the minimal resolution of the corresponding cusps $\left\{\bar{p}_{1}, \cdots, \bar{p}_{n}\right\}$ of $\Sigma_{\eta}$, and let $E_{i}+E_{i}^{\prime}$ be the exceptional curves over $\bar{p}_{i}$. Denote by $\tau_{\eta}$ the involution of $S_{\eta}$ induced by $\varphi_{\eta}$. Let $\delta$ be a divisor class on $S_{\eta}$.

Definition 0.2. The $n$ cusps $\left\{p_{1}, \cdots, p_{n}\right\}$ on $C$ are 3 -divisible of type $(\eta, \delta)$ if

(1) $C$ is an even divisor of type $\eta$, i.e., $C \equiv 2 \eta$.

(2) The cusps $\left\{\bar{p}_{1}, \cdots, \bar{p}_{n}\right\}$ of $\Sigma_{\eta}$ are 3-divisible of type $\delta$, namely there are some positive integers $a_{i}$ and $a_{i}^{\prime}$ with $a_{i}+a_{i}^{\prime}=3$ such that $\sum_{i=1}^{n}\left(a_{i} E_{i}+\right.$ $\left.a_{i}^{\prime} E_{i}^{\prime}\right)$ is a 3 -divisible divisor of type $\delta$ on $S_{\eta}$, i.e.,

$$
\sum_{i=1}^{n}\left(a_{i} E_{i}+a_{i}^{\prime} E_{i}^{\prime}\right) \equiv 3 \delta .
$$

Furthermore, $\delta$ is said to be compatible with $\eta$ if

$$
\tau_{\eta}^{*} \delta+\delta \equiv \sum_{i=1}^{n}\left(E_{i}+E_{i}^{\prime}\right) .
$$

Note that $(0.1)$ implies that $a_{i}+a_{i}^{\prime} \equiv 0(\bmod 3)$, and the relation $(0.1)$ is determined uniquely by the divisor $3 \delta$. Namely, $a_{i}$ and $a_{i}^{\prime}$ are determined by $3 \delta$. 
One can prove that the $n$ cusps on $\Sigma_{\eta}$ are 3 -divisible iff there is a smooth cyclic triple cover $\widetilde{\pi}: \widetilde{Y} \rightarrow \Sigma_{\eta}$ ramified exactly over the $n$ cusps. (Lemma 3.1).

For a generic triple cover $\pi: Y \rightarrow X$, it is easy to know that the branch locus has only cusps as its singularities. The trace free sheaf $\mathscr{E}_{\pi}$ is a rank two vector bundle,

$$
\pi_{*}\left(\mathscr{O}_{Y}\right)=\mathscr{O}_{X} \oplus \mathscr{E}_{\pi}
$$

Throughout this paper, we identify the first Chern class $c_{1}(\mathscr{E})$ of a vector bundle $\mathscr{E}$ with the divisor corresponding to its determinant $\operatorname{det} \mathscr{E}$.

Definition 0.3. Denote by $D_{2}$ the branch curves of a triple cover $\pi: Y \rightarrow X$ over which $\pi$ is totally ramified. We call the divisor class $\eta=-D_{2}-c_{1}\left(\mathscr{E}_{\pi}\right)$ the Galois invariant of the triple cover $\pi$.

The following are our main results.

Theorem 0.4. Let $\pi: Y \rightarrow X$ be a generic triple cover. Then the branch curve $C$ of $\pi$ is an even divisor of type $\eta=-c_{1}\left(\mathscr{E}_{\pi}\right)$, and the number $n$ of the cusps on $C$ is $3 c_{2}\left(\mathscr{E}_{\pi}\right)$. We have

$$
c_{1}^{2}\left(\mathscr{E}_{\pi}\right) \leq \frac{9}{2} c_{2}\left(\mathscr{E}_{\pi}\right), \quad \text { equivalently, } \quad n \geq \frac{1}{6} C^{2} .
$$

The equality holds if and only if $\mathscr{E}_{\pi}=\mathscr{L}^{-1} \oplus \mathscr{L}^{-2}$ for some line bundle $\mathscr{L}$, if and only if $C$ is defined by $f^{3}+g^{2}=0$, and the curves of $f$ and $g$ meet transversely.

For triple planes, the lower bound on the number of cusps is obtained by Bronowski ([2], II $\S 7$ ).

Theorem 0.5. Assume that $C$ is an irreducible curve with $n$ cusps, and as a divisor $C$ is even. Then $C$ is the branch locus of a generic triple cover with Galois invariant $\eta$ if and only if the $n$ cusps are 3-divisible of type $(\eta, \delta)$ and $\delta$ is compatible with $\eta$. Furthermore, this correspondence is one to one.

If $X=\mathbb{P}^{2}$, then $C$ is the branch curve of a triple plane if and only if the cusps are 3-divisible. This is a natural generalization of Zariski's characterization on the branched sextics with $n=6$ cusps.

Theorem 0.6. Assume that either the number of cusps of $C$ is less than $\frac{1}{3} C^{2}$, or $K_{X}+\frac{1}{2} C$ is nef, and

$$
C^{2}-6 C K_{X}>12\left(3 c_{2}(X)-c_{1}^{2}(X)\right) .
$$


Suppose that $C$ is the branch curve of a generic triple cover with Galois invariant $\eta$. Let $\mathscr{T}_{\eta} \subset \mathrm{Pic}\left(\mathrm{S}_{\eta}\right)$ be the subgroup consists of 3 -torsion classes $\varepsilon$ with $\tau_{\eta}^{*} \varepsilon \equiv-\varepsilon$. Let $\left|\mathscr{T}_{\eta}\right|$ be its order. Then there are exactly $\left|\mathscr{T}_{\eta}\right|$ generic triple covers ramified over $C$ with $\eta$ as their Galois invariants.

Note that if $X=\mathbb{P}^{2}$, then the numerical assumptions in this theorem are satisfied automatically. $\eta$ is determined by $C$. Because $S_{\tau}$ is simply connected in this case ([9], p.34, Corollary), $\left|\mathscr{T}_{\eta}\right|=1$. Hence we have

Corollary 0.7. Any triple plane is determined uniquely by its branch curve.

Theorem 0.8. Let $\mathscr{E}$ be a rank two vector bundle on $X$ with Chern classes $c_{1}(\mathscr{E})$ and $c_{2}(\mathscr{E})$, and let $\mathscr{H}$ be an ample line bundle. Then there is a number $m_{0}$ such that if $m>m_{0}$, then $\mathscr{E} \otimes \mathscr{H}^{-m}$ is the trace free sheaf of a generic triple cover over $X$. The divisor class of the branch curve $C$ and the number $n$ of cusps are as follows.

$$
C \equiv 2\left(2 m c_{1}(\mathscr{H})-c_{1}(\mathscr{E})\right), \quad n=3\left(c_{2}(\mathscr{E})-c_{1}(\mathscr{E}) c_{1}(\mathscr{H}) m+c_{1}^{2}(\mathscr{H}) m^{2}\right)
$$

If $\mathscr{E}$ is semistable, then $m_{0}$ depends only on $c_{1}(\mathscr{E}), c_{2}(\mathscr{E}), X$ and $\mathscr{H}$. In the second part of this paper, we will determine $\mathscr{T}_{\eta}$ when the branch curve $C$ is ample. We use the moduli space of stable rank two vector bundles to understand the structure of the varieties of the cuspidal curves with a 3-divisible set. Conversely, we will use the fundamental groups of cuspidal curves to study the associated rank two vector bundles and give some applications.

Acknowledgements: The author would like to thank professor Mina Teicher for introducing him to the interesting classical problem on cuspidal curves. He thanks also Prof. Jun Li for very useful discussions and suggestions about vector bundles, and the referee for valuable suggestions for the correction of the original manuscript.

\section{Preliminaries}

In this section, I will recall some basic facts on triple covers. Miranda [14] proved that 
Theorem 1.1. Any triple cover $\pi: Y \rightarrow X$ with a fixed trace free subsheaf $\mathscr{E}$ of $\pi_{*} \mathscr{O}_{Y}$ can be constructed by a non zero section s of $H^{0}\left(X, S^{3} \mathscr{E}^{\vee} \otimes \operatorname{det} \mathscr{E}\right) \cong$ $H^{0}\left(X, S^{3} \mathscr{E} \otimes \operatorname{det} \mathscr{E}^{-2}\right)$.

For any nonzero constant number $c \in \mathbb{C}, c s$ and $s$ define the same triple cover over $X$. Thus we can view the triple cover data $s$ with fixed $\mathscr{E}$ as an element in the projective space $P\left(H^{0}\left(X, S^{3} \mathscr{E}^{\vee} \otimes \operatorname{det} \mathscr{E}\right)\right)$. The corresponding triple cover is denoted by $\pi(s): Y(s) \rightarrow X$. $(\mathscr{E}, s)$ is called the triple cover data of $\pi(s)$.

$s$ can be viewed as a binary cubic form (see [19]), because locally

$$
s=\left(f_{0} x_{1}^{3}+3 f_{1} x_{1}^{2} x_{2}+3 f_{2} x_{1} x_{2}^{2}+f_{3} x_{2}^{3}\right) \otimes\left(x_{1}^{*} \wedge x_{2}^{*}\right) .
$$

For a cubic form $f=f_{0} x_{1}^{3}+3 f_{1} x_{1}^{2} x_{2}+3 f_{2} x_{1} x_{2}^{2}+f_{3} x_{2}^{3}$, we have three basic (co)invariants.

(1) The Hessian $H(f)$ of $f$ :

$$
H(f)=\frac{1}{36}\left(\frac{\partial^{2} f}{\partial x_{1}^{2}} \frac{\partial^{2} f}{\partial x_{2}^{2}}-\frac{\partial^{2} f}{\partial x_{1} \partial x_{2}} \frac{\partial^{2} f}{\partial x_{2} \partial x_{1}}\right)=h_{0} x_{1}^{2}+h_{1} x_{1} x_{2}+h_{2} x_{2}^{2},
$$

where

$$
h_{0}=f_{0} f_{2}-f_{1}^{2}, \quad h_{1}=f_{0} f_{3}-f_{1} f_{2}, \quad h_{2}=f_{1} f_{3}-f_{2}^{2} .
$$

$H$ induces an algebraic map (nonlinear)

$$
H: S^{3} \mathscr{E} \otimes(\operatorname{det} \mathscr{E})^{-2} \rightarrow S^{2} \mathscr{E} \otimes(\operatorname{det} \mathscr{E})^{-2} .
$$

(2) The discriminant $D(f)$ of $f: D(f)=-\frac{1}{27} \operatorname{disc}(f)=h_{1}^{2}-4 h_{0} h_{2}$.

$$
D: S^{3} \mathscr{E} \otimes(\operatorname{det} \mathscr{E})^{-2} \rightarrow(\operatorname{det} \mathscr{E})^{-2}
$$

(3) The Jacobian $J(f)$ of $f$ :

$$
J(f)=\frac{1}{27}\left(\frac{\partial f}{\partial x_{1}} \frac{\partial H}{\partial x_{2}}-\frac{\partial f}{\partial x_{2}} \frac{\partial H}{\partial x_{1}}\right)=\bar{f}_{0} x_{1}^{3}+3 \bar{f}_{1} x_{1}^{2} x_{2}+3 \bar{f}_{2} x_{1} x_{2}^{2}+\bar{f}_{3} x_{2}^{3},
$$

where

$$
\begin{aligned}
& \bar{f}_{0}=f_{0}^{2} f_{3}-3 f_{0} f_{1} f_{2}+2 f_{1}^{3}, \quad \bar{f}_{1}=f_{0} f_{1} f_{3}-2 f_{0} f_{2}^{2}+f_{1}^{2} f_{2}, \\
& \bar{f}_{2}=2 f_{1}^{2} f_{3}-f_{1} f_{2}^{2}-f_{2} f_{0} f_{3}, \quad \bar{f}_{3}=3 f_{2} f_{1} f_{3}-2 f_{2}^{3}-f_{3}^{2} f_{0} .
\end{aligned}
$$

$$
J: S^{3} \mathscr{E} \otimes(\operatorname{det} \mathscr{E})^{-2} \rightarrow S^{3} \mathscr{E} \otimes(\operatorname{det} \mathscr{E})^{-3}
$$


There is a syzygy among the invariants,

$$
4 H(f)^{3}+J(f)^{2}=D(f) f^{2} .
$$

$D(f)(x)=0$ iff $\pi$ is ramified over $x . H(f)(x)=0$ iff $\pi$ is totally ramified over $x$.

Because the invariants are independent of the choice of the base, we identify $f$ with $s$.

Two triple cover data $\left(\mathscr{E}_{1}, f_{1}\right)$ and $\left(\mathscr{E}_{2}, f_{2}\right)$ are said to be equivalent if there is an isomorphism $\tau: \mathscr{E}_{1} \cong \mathscr{E}_{2}$ such that the induced isomorphism $S^{3} \tau \otimes \operatorname{det} \tau^{-2}$ from $S^{3} \mathscr{E}_{1} \otimes \operatorname{det} \mathscr{E}_{1}^{-2}$ to $S^{3} \mathscr{E}_{2} \otimes \operatorname{det} \mathscr{E}_{2}^{-2}$ maps $f_{1}$ to $f_{2}$

Two finite covers $\pi_{i}: Y_{i} \rightarrow X_{i}(i=1,2)$ are said to be equivalent if there are isomorphisms $\psi_{1}: X_{2} \rightarrow X_{1}$ and $\psi_{2}: Y_{2} \rightarrow Y_{1}$ such that $\psi_{1} \circ \pi_{2}=\pi_{1} \circ \psi_{2}$, we denote it by $\pi_{1} \sim \pi_{2}$. If we consider finite covers over a fixed surface $X_{2}=X_{1}=$ $X$, we always assume that $\psi_{1}$ is the identity.
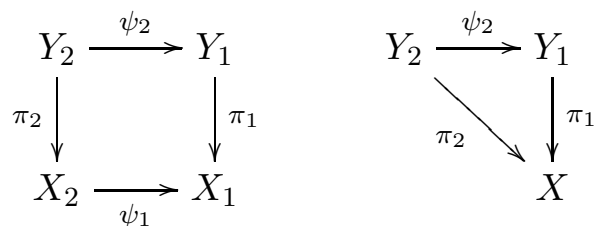

Theorem 1.2. There is a one to one correspondence between the two sets of equivalent classes.

$\{$ Triple cover data $(\mathscr{E}, f)$ on $X\} / \sim \longleftrightarrow\{$ Triple covers $\pi: Y \rightarrow X\} / \sim$

Now let $\pi: Y \rightarrow X$ be a normal triple cover with a trace-free subsheaf $\mathscr{E}$, and let $\mathscr{L}^{\vee} \subset \mathscr{E}$ be any maximal invertible subsheaf. It is well-known that there is a short exact sequence

$$
0 \rightarrow \mathscr{L}^{\vee} \rightarrow \mathscr{E} \rightarrow \mathscr{I}_{\Delta} \otimes \mathscr{M}^{\vee} \rightarrow 0
$$

where $\Delta$ is a zero-dimensional subscheme of $X$ and $\mathscr{M}$ is an invertible sheaf. If $z$ is a local base of the image of $\mathscr{L}^{\vee}$ in $\mathscr{E}$ and $x_{1}=z^{*}$ is the corresponding base of $z$ in $\mathscr{E}^{\vee}$, then the coefficient $f_{0}$ is a global section of $\mathscr{L}^{2} \otimes \mathscr{M}^{\vee}$. This gives a homomorphism

$$
I_{0}: S^{3} \mathscr{E} \otimes \operatorname{det} \mathscr{E}^{-2} \longrightarrow \mathscr{L}^{2} \otimes \mathscr{M}^{\vee}, \quad f \mapsto f_{0} .
$$


Similarly,

$$
\begin{aligned}
& H_{0}: S^{2} \mathscr{E} \otimes \operatorname{det} \mathscr{E}^{-2} \longrightarrow \mathscr{L}^{2}, h_{0} x_{1}^{2}+h_{1} x_{1} x_{2}+h_{2} x_{2}^{2} \mapsto h_{0} . \\
& J_{0}: S^{3} \mathscr{E} \otimes \operatorname{det} \mathscr{E}^{-3} \longrightarrow \mathscr{L}^{3}, \quad \bar{f} \mapsto \bar{f}_{0} .
\end{aligned}
$$

The syzygy implies

$$
4 h_{0}^{3}+\bar{f}_{0}^{2}=D(f) f_{0}^{2}
$$

In $[18,19]$, we proved that this invertible subsheaf $\mathscr{L}^{\vee}$ determines a unique cubic equation defining the triple cover,

$$
z^{3}+a_{1} a_{2}^{2} b_{1} a_{0} z+a_{1} a_{2}^{2} b_{1}^{2} b_{0}=0,
$$

where $z$ is the fiber coordinate of $\mathscr{L}$ and the coefficients are respectively two global sections of $\mathscr{L}^{2}$ and $\mathscr{L}^{3}, a_{1}, a_{2}$ and $b_{1}$ are square free, and $a=4 a_{1} a_{2}^{2} a_{0}^{3}$ and $b=27 b_{1} b_{0}^{2}$ are coprime. More precisely, $Y$ is the normalization of the surface $Y_{0} \subset \mathscr{L}$ defined by the cubic equation.

The cubic defining equation is equivalent to

$$
z^{3}+3 h_{0} z-\bar{f}_{0}=0
$$

So

$$
3 h_{0}=a_{1} a_{2}^{2} b_{1} a_{0}, \quad-\bar{f}_{0}=a_{1} a_{2}^{2} b_{1}^{2} b_{0} .
$$

Let $c:=a+b$. Suppose $c$ has a factorization $c=c_{1} c_{0}^{2}$, where $c_{1}$ is square free. We have (see [19], §3.3)

$$
f_{0}=a_{2} b_{1} c_{0}
$$

We use the capital letter $A_{i}, B_{i} \cdots$ to denote the divisors of the corresponding sections $a_{i}, b_{i}, \cdots$.

Theorem 1.3. The Galois invariant of $\pi$ is $\eta=-A_{1}-A_{2}-c_{1}(\mathscr{E})$.

(1) $\pi$ is totally (resp. simply) ramified over $A_{1}+A_{2}$ (resp. $B_{1}+C_{1}$ ).

(2) $2 A_{1}+2 A_{2}+B_{1}+B_{2} \equiv-2 c_{1}(\mathscr{E})$, i.e., $B_{1}+C_{1} \equiv 2 \eta$ is an even divisor.

(3) The non-normal locus in $X$ of the surface $Y_{0}$ is $A_{2}+B_{1}+C_{0}$, which is linearly equivalent to $2 L-M$, where $\mathscr{L}=\mathscr{O}_{X}(L)$ and $\mathscr{M}=\mathscr{O}_{X}(M)$.

(4) $\pi$ is Galois if and only if $\pi$ is totally ramified over the branch locus and $\eta \equiv 0$. Namely, $B_{1}=C_{1}=0$ and $\eta \equiv 0$. (If $X$ is projective, then $\eta \equiv 0$ implies $B_{1}=C_{1}=0$ ). 
Cuspidal Curves and Vector Bundles on an Algebraic Surface, I 1793

(5) Let $\varphi: \Sigma \rightarrow X$ be the canonical double cover ramified over $B_{1}+C_{1}$ determined by $\eta$, and let $\widetilde{\pi}: \widetilde{Y} \rightarrow \Sigma$ be the canonical pullback triple cover of $\pi$ under the base change $\varphi$.

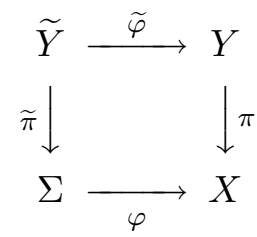

Then $\widetilde{\pi}$ is a cyclic triple cover ramified over $\varphi^{*}\left(A_{1}+A_{2}\right)$ and some isolated points coming from the points on $B_{1}+C_{1}$ where $\pi$ is totally ramified.

(6) (Uniqueness) Let $\varphi^{\prime}: \Sigma^{\prime} \rightarrow X$ be any double cover ramified over $B_{1}+C_{1}$ such that the pullback triple cover $\widetilde{\pi}^{\prime}: \tilde{Y}^{\prime} \rightarrow \Sigma^{\prime}$ is a cyclic triple cover. If $\pi$ is not Galois, then $\varphi^{\prime}$ is isomorphic to $\varphi$.

Sketch of the Proof. One can find the details of the proof of (1) to (4) in $[18,19,21]$.

In order to prove (5), we need to compute $\widetilde{\pi}_{*} \mathscr{O}_{\widetilde{Y}}$, where $\widetilde{Y}$ is the normalization of $Z=\Sigma \times_{X} Y$.

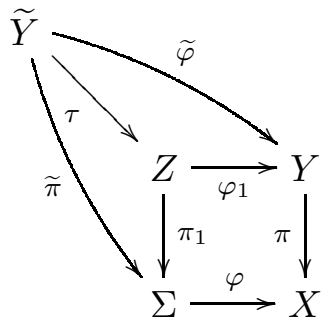

Let $U$ be the open set of $X$ over which $Y$ and $\Sigma$ are smooth. Because $Y$ and $\Sigma$ are normal, they have at most a finite number singular points. Thus $X \backslash U$ consists of a finite number of points. We only need to prove (5) and (6) for $X=U$. Namely, we can assume that $Y$ and $\Sigma$ are smooth.

Note that $\pi^{*}\left(B_{1}+C_{1}\right)=2 R+D$. So the pullback double cover is ramified over $2 R+D$ and $Z$ is non-normal along $\hat{R}$ over $R$. From the computation of the normalization, we have

$$
0 \rightarrow \mathscr{O}_{Z} \rightarrow \tau_{*} \mathscr{O}_{\widetilde{Y}} \rightarrow \mathscr{O}_{\hat{R}} \rightarrow 0
$$

Because $R^{1} \pi_{1 *} \mathscr{O}_{Z}=0$, we have

$$
0 \rightarrow \pi_{1 *} \mathscr{O}_{Z} \rightarrow \widetilde{\pi}_{*} \mathscr{O}_{\widetilde{Y}} \rightarrow \pi_{1 *} \mathscr{O}_{\hat{R}} \rightarrow 0,
$$


$\varphi$ is flat, by the commutativity of cohomology with flat base changes, we get

$$
\pi_{1 *} \mathscr{O}_{Z}=\pi_{1 *} \varphi_{1}^{*} \mathscr{O}_{Y}=\varphi^{*} \pi_{*} \mathscr{O}_{Y}=\mathscr{O}_{\Sigma} \oplus \varphi^{*} \mathscr{E}
$$

Let $\bar{R}=\pi_{1}(\hat{R}) . \pi_{1}$ maps $\hat{R}$ birationally to $\bar{R}$, and $2 \bar{R}=\varphi^{*}\left(B_{1}+C_{1}\right)$, we obtain

$$
0 \rightarrow \mathscr{O}_{\Sigma} \oplus \varphi^{*} \mathscr{E} \rightarrow \mathscr{O}_{\Sigma} \oplus \mathscr{E}_{\tilde{\pi}} \rightarrow \mathscr{O}_{\bar{R}} \rightarrow 0
$$

Namely,

$$
0 \rightarrow \varphi^{*} \mathscr{E} \rightarrow \mathscr{E}_{\pi} \rightarrow \mathscr{O}_{\bar{R}} \rightarrow 0
$$

Hence $\varphi^{*} \mathscr{E}$ is the elementary modification of $\mathscr{E} \widetilde{\pi}$ along $\bar{R}([8]$, p.41), and

$$
c_{1}\left(\mathscr{E}_{\widetilde{\pi}}\right)=c_{1}\left(\varphi^{*} \mathscr{E}\right)+\bar{R}=\varphi^{*}\left(c_{1}(\mathscr{E})\right)+\varphi^{*} \eta=-\varphi^{*}\left(A_{1}+A_{2}\right),
$$

so the Galois invariant of $\widetilde{\pi}$ is zero. Therefore, $\widetilde{\pi}$ is Galois.

Finally, suppose $\varphi^{\prime}$ is determined by $\eta^{\prime}$ such that $B_{1}+C_{1} \equiv 2 \eta^{\prime}$. We will prove that $\eta^{\prime} \equiv \eta$ under the condition of (6). Thus $\varphi^{\prime}$ and $\varphi$ are equivalent.

With the same computation as above, we have

$$
c_{1}\left(\mathscr{E}_{\widetilde{\pi}^{\prime}}\right)=c_{1}\left(\varphi^{*} \mathscr{E}\right)+\bar{R}^{\prime}=\varphi^{\prime *}\left(c_{1}(\mathscr{E})\right)+\varphi^{*} \eta^{\prime},
$$

by the Galoisness of $\widetilde{\pi}^{\prime}$, we have $-\varphi^{* *}\left(A_{1}+A_{2}\right) \equiv c_{1}\left(\mathscr{E}_{\tilde{\pi}^{\prime}}\right)$, so $\varphi^{\prime *}\left(\eta^{\prime}-\eta\right) \equiv 0$.

Note that $\varphi_{*}^{\prime} \varphi^{*} \mathscr{O}_{\Sigma}\left(\eta^{\prime}-\eta\right)=\mathscr{O}_{X}\left(\eta^{\prime}-\eta\right) \oplus \mathscr{O}_{X}(-\eta)$, and

$$
H^{0}\left(\varphi_{*}^{\prime} \varphi^{*} \mathscr{O}_{\Sigma}\left(\eta^{\prime}-\eta\right)\right)=H^{0}\left(\mathscr{O}_{\Sigma}\left(\varphi^{\prime *}\left(\eta^{\prime}-\eta\right)\right)=H^{0}\left(\mathscr{O}_{\Sigma}\right)=\mathbb{C}\right.
$$

because $\pi$ is not Galois, $H^{0}\left(\mathscr{O}_{X}(-\eta)\right)=0$. We get $H^{0}\left(\mathscr{O}_{X}\left(\eta^{\prime}-\eta\right)\right)=\mathbb{C}$. On the other hand, $\eta^{\prime}-\eta$ is a torsion divisor, so it must be zero, i.e., $\eta^{\prime} \equiv \eta$.

Corollary 1.4. Let $\pi: Y \rightarrow X$ be a generic triple cover ramified over $C$. Then there is a unique base change $\varphi: \Sigma \rightarrow X$ of degree two ramified over $C$ such that the pullback triple cover $\widetilde{\pi}: \widetilde{Y} \rightarrow \Sigma$ is a smooth cyclic triple cover ramified exactly over the cusps on $\Sigma$.

Proof. We only need to note that $\widetilde{\varphi}$ is a double cover ramified over a smooth curve $D$, where $\pi^{*} C=2 R+D$, so $\widetilde{Y}$ is smooth.

Corollary 1.5. Suppose that $\pi$ is not Galois, and let $\sigma$ (resp. $\tau$ ) be the automorphism of $\widetilde{Y}$ induced by $\widetilde{\pi}$ (resp. $\widetilde{\varphi}$ ). Then

$$
\tau^{2}=\sigma^{3}=1, \quad \tau \sigma \tau^{-1}=\sigma^{-1} .
$$


Thus the composition map $\tilde{Y} \rightarrow X$ is a Galois cover with group $G=<\tau, \sigma>\cong S_{3}$, which is the Galois closure of $\pi$. Conversely, $\pi: Y \rightarrow X$ can be reconstructed from $\tilde{Y} \rightarrow X$, i.e., $Y=\tilde{Y} /<1, \tau>$ and $\pi$ is induced by $\widetilde{Y} \rightarrow X$.

Proof. Note that $\tau$ is the lift of the involution $\tau_{0}$ on $\Sigma$ induced by $\varphi$, so $\tilde{\pi} \tau=\tau_{0} \tilde{\pi}$. On the other hand, $\sigma$ is induced by $\tilde{\pi}$, hence we have $\tilde{\pi} \sigma=\widetilde{\pi}$. Then we get

$$
\tilde{\pi} \tau \sigma \tau^{-1}=\tau_{0} \tilde{\pi} \sigma \tau=\tau_{o} \tilde{\pi} \tau=\tau_{0}^{2} \tilde{\pi}=\tilde{\pi},
$$

which implies $\tau \sigma \tau^{-1}$ is also an automorphism of $\widetilde{Y}$ induced by $\widetilde{\pi}$. Thus $\tau \sigma \tau^{-1}=$ $\sigma^{m}$ for $m=1$ or -1 .

If $m=1$, then $\tau$ commutes with $\sigma$. It is easy to see that $\tau$ induces an automorphism $\widetilde{\sigma}$ of $Y=\widetilde{Y} /<1, \tau>$ of order 3. Hence $\widetilde{\varphi} \sigma=\widetilde{\sigma} \widetilde{\varphi}$. On the other hand, $\widetilde{\pi} \sigma=\widetilde{\pi}$, we get $\varphi \widetilde{\pi} \sigma=\varphi \widetilde{\pi}$, i.e., $\pi \widetilde{\varphi} \sigma=\pi \widetilde{\varphi}$. Then we obtain $\pi \widetilde{\sigma} \widetilde{\varphi}=\pi \widetilde{\varphi}$. Because $\widetilde{\varphi}$ is surjective, we have $\pi \widetilde{\sigma}=\pi$. This means that $\pi$ is a cyclic triple cover, a contradiction. Hence $m=-1$. This proves the lemma.

\section{Proof of Theorem 0.4}

Lemma 2.1. (Bogomolov's inequality [1]) Let $\mathscr{E}$ be a rank two vector bundle over $X$, and let $c_{1}$ and $c_{2}$ be its Chern classes. If $c_{1}^{2}>4 c_{2}$, then there exists a maximal invertible subsheaf $\mathscr{L}^{\vee}=\mathscr{O}_{X}(-L) \subset \mathscr{E}$ such that for any nef and big divisor $H$ we have

(1) $\left(-2 L-c_{1}\right) H>0$,

(2) $\left(-2 L-c_{1}\right)^{2} \geq c_{1}^{2}-4 c_{2}$.

Lemma 2.2. If $\mathscr{E}=\mathscr{E}_{\pi}$ for some generic triple cover $\pi: Y \rightarrow X$, and $\mathscr{L}^{\vee}=$ $\mathscr{O}_{X}(-L)$ is a maximal invertible subsheaf of $\mathscr{E}$, then

$$
3 L+c_{1} \equiv C_{0}:=\operatorname{div}\left(c_{0}\right) .
$$

Hence for any nef and big divisor $H$,

$$
L H \geq-\frac{c_{1} H}{3} .
$$

Proof of Theorem 0.4. Note first that $3 c_{2}=n>0$, so we can assume that $c_{1}^{2}>0$, i.e., $C^{2}>0$. Because the branch locus $C$ is irreducible, $C$ is nef, i.e., for any curve $\Gamma, \Gamma C \geq 0$. We can assume also that $c_{1}^{2}>4 c_{2}$. 
By Lemma 2.1, there is a divisor $L$ satisfies (1) and (2) in this lemma. Hence we can find respectively two global sections $a_{0}$ and $b_{0}$ of $\mathscr{L}^{2}$ and $\mathscr{L}^{3}$ such that the defining equation of the generic triple cover $\pi$ is $z^{3}+a_{0} z+b_{0}=0$. Note that we have $4 a_{0}^{3}+27 b_{0}^{2}=c_{1} c_{0}^{2}$.

Now by Hodge index theorem and Lemma 3.1,

$$
\left(\left(-2 L-c_{1}\right) H\right)^{2} \geq\left(-2 L-c_{1}\right)^{2} H^{2} \geq\left(c_{1}^{2}-4 c_{2}\right) H^{2} .
$$

Because $\left(-2 L-c_{1}\right) H>0$, we have

$$
\left(-2 L-c_{1}\right) H \geq \sqrt{c_{1}^{2}-4 c_{2}} \sqrt{H^{2}}
$$

i.e.,

$$
L H \leq-\frac{1}{2} c_{1} H-\frac{1}{2} \sqrt{H^{2}} \sqrt{c_{1}^{2}-4 c_{2}}
$$

On the other hand, by Lemma 3.2,

$$
L H \geq-\frac{1}{3} c_{1} H .
$$

Combining (2.1) and (2.2), we obtain

$$
\frac{1}{3} c_{1} H \geq \frac{1}{2} c_{1} H+\frac{1}{2} \sqrt{H^{2}} \sqrt{c_{1}^{2}-4 c_{2}} .
$$

Because $-c_{1}$ is nef and big, let $H=-c_{1}$, then we get

$$
-\frac{1}{3} c_{1}^{2} \geq-\frac{1}{2} c_{1}^{2}+\frac{1}{2} \sqrt{c_{1}^{2}} \sqrt{c_{1}^{2}-4 c_{2}}
$$

i.e.,

$$
c_{1}^{2} \leq \frac{9}{2} c_{2}
$$

The equality in (2.4) implies that all of the above inequalities become equalities. In particular, the first equality is

$$
\left(\left(-2 L-c_{1}\right)\left(-c_{1}\right)\right)^{2}=\left(-2 L-c_{1}\right)^{2}\left(-c_{1}\right)^{2} .
$$

By Hodge index theorem, we see that $-2 L-c_{1}$ is numerically equivalent to multiple of $-c_{1}$, hence $L \sim \lambda c_{1}$. The equality in (2.2) implies $\lambda=-1 / 3$, thus $C_{0} \equiv 3 L+c_{1} \sim 0$. Because $X$ is projective, $C_{0}=0$. Hence $c_{0}$ is a non zero constant, i.e., the non normal locus is empty. Therefore, $\mathscr{E}_{\pi}=\mathscr{O}_{X}(-L) \oplus \mathscr{O}_{X}(-2 L)$, and the branch locus is defined by $4 a_{0}^{3}+27 b_{0}^{2}=0$. Since $a_{0}$ and $b_{0}$ are respectively the global sections of $\mathscr{O}_{X}(2 L)$ and $\mathscr{O}_{X}(3 L)$, and the divisors of $a_{0}$ and $b_{0}$ pass 
through the $n=\frac{2}{3} c_{1}^{2}=(2 L) \cdot(3 L)$ cusps of the branch locus, we can see easily that the divisors of $a_{0}$ and $b_{0}$ meet normally.

It is obvious that the converse is also true.

Remark 2.3. If the branch locus $C$ has other rational double points, but $\pi$ is not totally ramified over them, then the above inequality still holds. In this case, $X$ has singularities.

\section{Proof of Theorem 0.5}

Let $C$ be an irreducible curve on $X$ with only $n \geq 1$ cusps as its singularity. Assume that $C$ is an even divisor. Denote by $\varphi: \Sigma \rightarrow X$ the double cover ramified over $C$. Then $\Sigma$ has $n$ cusps $p_{1}, \cdots, p_{n}$ of type $A_{2}$ coming from those on $C$. Denote by $\varepsilon: S \rightarrow \Sigma$ the minimal resolution of the cusps, and denote by $E_{i}+E_{i}^{\prime}$ the exceptional set over $p_{i}$. Then

Lemma 3.1. The $n$ cusps on $\Sigma$ are 3-divisible of type $\delta$ if and only if there is a smooth cyclic triple cover $\widetilde{\pi}: \widetilde{Y} \rightarrow \Sigma$ ramified exactly over the cusps and determined by $\delta$.

Proof. Consider the fiber product of $\widetilde{\pi}$ with $\varepsilon$, we see that there is a cyclic triple cover $\bar{\pi}: \bar{S} \rightarrow S$ over $S$ ramified over the exceptional curves.

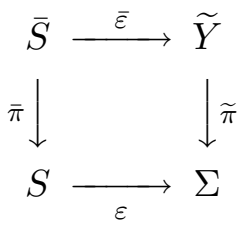

Thus there are two divisors $L$ and $M$, as well as two reduced divisors $\mathscr{B}$ and $\mathscr{C}$ such that the support $\mathscr{B}+\mathscr{C}$ is the divisor of the $n A_{2}$ curves, and

$$
\mathscr{B} \equiv 2 M-L, \quad \mathscr{C} \equiv 2 L-M .
$$

So $\mathscr{B}+2 \mathscr{C} \equiv 3 L$, by considering the intersection numbers of both sides with $E_{i}$ or $E_{i}^{\prime}$, we see that $E_{i}$ and $E_{i}^{\prime}$ can not be contained simultaneously in the same curve $\mathscr{B}$ or $\mathscr{C}$. Thus we can assume that $\mathscr{B}=\sum_{i=1}^{n} E_{i}$ and $\mathscr{C}=\sum_{i=1}^{n} E_{i}^{\prime}$. Then the cusps are 3 -divisible by definition.

Conversely, suppose we have (0.1). Consider the blowing ups $\varepsilon^{\prime}: S^{\prime} \rightarrow S$ of $S$ at the $n$ points $E_{i} \cap E_{i}^{\prime}$, and denote by $\bar{E}_{i}$ and $\bar{E}_{i}^{\prime}$ the strict transforms of $E_{i}$ and 
$E_{i}^{\prime}$ respectively. Then we have

$$
\sum_{i=1}^{n}\left(\bar{E}_{i}+2 \bar{E}_{i}^{\prime}\right) \equiv 3 \delta^{\prime}
$$

for some divisor $\delta^{\prime}$ on $S^{\prime}$. Thus there is a smooth cyclic triple cover $\widetilde{S} \rightarrow S^{\prime}$ ramified over these $(-3)$-curves $\sum_{i=1}^{n}\left(\bar{E}_{i}+\bar{E}_{i}^{\prime}\right)$. It is easy to know that the preimages of $\bar{E}_{i}$ and $\bar{E}_{i}^{\prime}$ in $\widetilde{S}$ are $(-1)$-curves, and the preimages of the exceptional curves of $\varepsilon^{\prime}$ on $\widetilde{S}$ are $(-3)$-curves. All of these curves on $\widetilde{S}$ can be contracted to smooth points of a surface $\widetilde{Y}$. The triple cover induces a smooth triple cover $\tilde{\pi}: \widetilde{Y} \rightarrow \Sigma$ ramified exactly over the cusps.

Theorem 3.2. Let $C$ be the branch curve of a generic triple cover $\pi: Y \rightarrow X$. Then the cusps of $C$ are 3 -divisible.

Proof. Consider the pullback cyclic triple cover $\widetilde{\pi}: \widetilde{Y} \rightarrow \Sigma$ given in Sect. 1. We have seen that when $\pi: Y \rightarrow X$ is a generic triple cover, $\widetilde{\pi}: \widetilde{Y} \rightarrow \Sigma$ is a smooth cyclic triple cover ramified exactly over the cusps on $\Sigma$. Thus, the $n$ cusps on $\Sigma$ are 3 divisible. By definition, the $n$ cusps on $C$ are 3 divisible.

In what follows, we always assume that $C$ is an even irreducible cuspidal curve whose cusps are 3-divisible of type $(\eta, \delta)$.

By Lemma 3.1, we have a smooth finite cover $\Pi: \widetilde{Y} \rightarrow X$ of degree 6 ramified over $C$.

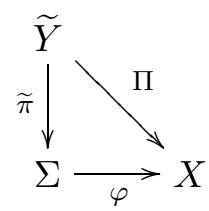

Lemma 3.3. Denote by $\sigma$ (resp. $\tau_{\eta}$ ) the automorphism induced by $\widetilde{\pi}$ (resp. $\varphi$ ). The following statements are equivalent.

(1) $\Pi$ is Galois with group $G \cong S_{3}$.

(2) $\tau_{\eta}$ can be lifted to $\tilde{Y}$.

(3) $\delta$ is compatible with $\eta$, i.e.,

$$
\tau_{\eta}^{*} \delta+\delta \equiv \sum_{i=1}^{n}\left(E_{i}+E_{i}^{\prime}\right) .
$$


Proof. If $\Pi$ is Galois with group $G \cong S_{3}$, then the automorphism $\sigma$ of $\widetilde{Y}$ induced by $\tilde{\pi}$ generates a normal subgroup $N$ of $G$ of order 3 . Now it is easy to see that one element $\tau$ of order 2 of $G$ induces $\tau_{0}$. Conversely, let $\tau$ be the lift of $\tau_{0}$. Then $\Pi$ is a Galois cover of degree 6 . We claim that $\tau$ can not commute with $\sigma$. Otherwise, $\Pi$ is a cyclic cover ramified over $C$. The branch locus $C$ of $\Pi$ is irreducible, so $\Pi$ is defined locally by $z^{6}=f$, where $f$ is the local defining equation of $C$. Because $\widetilde{Y}$ is smooth, $C$ must be smooth, a contradiction. Hence the Galois group of $\Pi$ is $S_{3}$. Therefore, (1) is equivalent to (2).

Suppose that $\Pi$ is Galois with group

$$
G=\left\{\sigma, \tau \mid \sigma^{3}=1, \tau^{2}=1, \tau \sigma \tau=\sigma^{2}\right\} \cong S_{3} .
$$

$\widetilde{\pi}$ is the quotient map of $\widetilde{Y}$ under the action of $\sigma$. According to the eigen-space decomposition of $\mathscr{O}_{\bar{S}}$ under the action of $\sigma$, we have

$$
\bar{\pi}_{*} \mathscr{O}_{\bar{S}}=\mathscr{O}_{S} \oplus \mathscr{O}_{S}(-L) \oplus \mathscr{O}_{S}(-M)
$$

where $L=\delta$ and $M=-\delta+\sum_{i=1}^{n}\left(E_{i}+E_{i}^{\prime}\right)$. Because $\tau \sigma \tau=\sigma^{2}$, one can check that $\tau$ acts trivially on $\mathscr{O}_{S}$ and exchange the two eigen-spaces $\mathscr{O}_{\bar{S}}(-L)$ and $\mathscr{O}_{S}(-M)$. This implies $\tau_{\eta}^{*} L \equiv M$ and $\tau_{\eta}^{*} M \equiv L$.

Now we need to compute $L$ and $M$. Suppose the divisor $\sum_{i=1}^{n}\left(a_{i} E_{i}+a_{i}^{\prime} E_{i}^{\prime}\right)=$ $\mathscr{B}+2 \mathscr{C}=\operatorname{div}\left(b c^{2}\right)$ is defined by the global section $b c^{2}$, where $b \in H^{0}\left(\mathscr{O}_{S}(\mathscr{B})\right)$ (resp. $\left.c \in H^{0}\left(\mathscr{O}_{S}(\mathscr{C})\right)\right)$ the defining equation of $\mathscr{B}$ (resp. $\mathscr{C}$ ). Then the triple cover $\widetilde{\pi}$ is defined as the normalization of the cover defined by $z^{3}=b c^{2}$. The normalization is just to add a new element $w=z^{2} / c$. It is well-known that the three eigen-spaces are generated by $1, z$ and $w$ respectively. Hence we have $L \equiv \delta$ and $M \equiv 2 \delta-\operatorname{div}(c) \equiv-\delta+\operatorname{div}(b c) \equiv-\delta+\sum_{i=1}^{n}\left(E_{i}+E_{i}^{\prime}\right)$. So $\delta$ is compatible with $\eta$.

Finally, suppose (3) is true. With the notations above, the triple cover surface $\bar{S}$ is a subvariety of the vector bundle $[L] \oplus[M]$ defined by

$$
z^{2}=b w, \quad z w=b c, \quad w^{2}=b z .
$$

Because $\tau_{\eta}$ exchanges $E_{i}$ and $E_{i}^{\prime}$, we know that $\tau_{\eta}^{*} b=c$ and $\tau_{\eta}^{*} c=b$ for a proper choice of $b$ and $c$, for example, let $c=\tau_{\eta}^{*} b$. Similarly, the equation (3.2) induces isomorphisms between $\mathscr{O}_{S}(-L)$ and $\mathscr{O}_{S}(-M)$. So $\tau_{\eta}$ induces an automorphism $\tau$ of $\mathscr{E}=\mathscr{O}_{S} \oplus \mathscr{O}_{S}(-L) \oplus \mathscr{O}_{S}(-M)$. By a proper choice of $z$ and $w$, we can see 
that $\tau$ is compatible with the product (3.3). $\tau$ preserves the ring structure. The induced automorphism $\tau$ on $\bar{S}$ or $\widetilde{Y}$ is what we desired.

Lemma 3.4. Under the condition of Lemma 3.3, let $H$ be the subgroup of $G$ generated by an element $\tau$ of order 2 . Let $Y=\widetilde{Y} / H$ and $\widetilde{\varphi}: \widetilde{Y} \rightarrow Y$ be the quotient map. Then the induced map $\pi: Y \rightarrow X$ is a generic triple cover ramified over $C$ with Galois invariant $\eta$.

Proof. We only need to prove that $\pi$ is a generic triple cover. Because $\tilde{\pi}$ is unramified over a generic point of $\varphi^{-1}(C)$, we see that every component of $\Pi^{*} C$ has multiplicity 2 . So $\pi$ has no totally branched curve. $\Pi$ is unramified over $X \backslash C$, the branch locus $B_{\pi}$ of $\pi$ is contained in $C$. Because $X$ is smooth, $B_{\pi}$ is either empty or a curve. If $\pi$ is unramified, then $\widetilde{\varphi}$ is a double cover ramified over a cuspidal curve $\pi^{-1}(C)$, so $\tilde{Y}$ is singular, a contradiction. Hence $B_{\pi}=C$ and $\pi$ is simply ramified over $C$. Finally, because $\Pi$ is totally ramified over the cusps on $C$, so does $\pi$. Hence $Y$ is smooth ([14] or [18]). Now we know that $\pi$ is a generic triple cover.

By the uniqueness of the canonical base change, we know that the Galois invariant of $\pi$ must be $\eta$, i.e., $-c_{1}\left(\mathscr{E}_{\pi}\right) \equiv \eta$.

Lemma 3.5. Let $C$ be an irreducible cuspidal curve whose cusps are 3-divisible of type $\left(\eta, \delta^{\prime}\right)$. Then there exists a $\delta$ compatible with $\eta$ such that the cusps are 3-divisible of type $(\eta, \delta)$.

Proof. With the notations in the proof of the previous lemma, we assume that the 3 -divisibility is given by $\mathscr{B}+2 \mathscr{C} \equiv 3 \delta^{\prime}$. Then

$$
\bar{\pi}_{*} \mathscr{O}_{\bar{S}}=\mathscr{O}_{S} \oplus \mathscr{O}_{S}\left(-L^{\prime}\right) \oplus \mathscr{O}_{S}\left(-M^{\prime}\right),
$$

where $L^{\prime}=\delta^{\prime}$ and $M^{\prime}=2 \delta^{\prime}-\mathscr{C}$. We have $\mathscr{B} \equiv 2 M^{\prime}-L^{\prime}, \mathscr{C} \equiv 2 L^{\prime}-M^{\prime}$, or $\mathscr{B}+2 \mathscr{C} \equiv 3 L^{\prime}, 2 \mathscr{B}+\mathscr{C} \equiv 3 M^{\prime}$. Note that $\tau_{\eta}$ exchanges $E_{i}$ and $E_{i}^{\prime}$. Hence $\tau_{\eta}^{*} \mathscr{B}=\mathscr{C}$ and $\tau_{\eta}^{*} \mathscr{C}=\mathscr{B}$.

Now we claim that there exists a divisor $\delta$ such that $\mathscr{B}+2 \mathscr{C} \equiv 3 \delta$ and the cyclic triple cover determined by $\delta$ satisfies

$$
\tau_{\eta}^{*} \mathscr{B}=\mathscr{C}, \quad \tau_{\eta}^{*} \mathscr{C}=\mathscr{B}, \quad \tau_{\eta}^{*} L \equiv M, \quad \tau_{\eta}^{*} M \equiv L .
$$

Indeed, from $\mathscr{B}+2 \mathscr{C} \equiv 3 L^{\prime}$ and $2 \mathscr{B}+\mathscr{C} \equiv 3 M^{\prime}$, we get $3 \tau_{\eta}^{*} L^{\prime} \equiv 3 M^{\prime}$. Thus there is a 3 -torsion divisor $\varepsilon$ such that $\tau_{\eta}^{*} L^{\prime}=M^{\prime}+\varepsilon$, which implies $\tau_{\eta}^{*} M^{\prime}=$ 
$L^{\prime}-\tau_{\eta}^{*} \varepsilon$. Note that $\mathscr{B}+\mathscr{C} \equiv L^{\prime}+M^{\prime}$ is invariant under the action of $\tau_{\eta}^{*}$. Hence $\tau_{\eta}^{*} \varepsilon \equiv \varepsilon$. Let $\delta=\delta^{\prime}+\varepsilon$. Then we have $L=L^{\prime}+\varepsilon$, and $M=M^{\prime}-\varepsilon$. Then we can check that $(\mathscr{B}, \mathscr{C}, L, M)$ satisfies (3.4). The claim is proved.

Now we see that $\delta=\delta^{\prime}+\varepsilon$ is compatible with $\eta$ and $\mathscr{B}+2 \mathscr{C} \equiv 3 \delta$, i.e., the cusps are 3 -divisible of type $(\eta, \delta)$.

Lemma 3.6. With the notations above, let $\delta$ and $\delta^{\prime}$ be two divisors compatible with $\eta$. Then $\mathscr{B}+2 \mathscr{C} \equiv 3 \delta \equiv 3 \delta^{\prime}$ if and only if there is a 3 -torsion divisor $\varepsilon$ such that

$$
\delta^{\prime}=\delta+\varepsilon, \quad \tau_{\eta}^{*} \varepsilon \equiv-\varepsilon
$$

Proof. Let $\varepsilon=\delta^{\prime}-\delta$. So $3 \varepsilon=3 \delta^{\prime}-3 \delta \equiv 0$, and because $\delta^{\prime}$ and $\delta$ are compatible with $\eta$, we can check easily that $\tau_{\eta}^{*} \varepsilon \equiv-\varepsilon$. The converse is obvious.

Two generic cover $\pi_{1}: Y_{1} \rightarrow X$ and $\pi_{2}: Y_{2} \rightarrow X$ branched along $C$ are called equivalent, if there is an isomorphism $\psi: Y_{2} \rightarrow Y_{1}$ such that $\pi_{2}=\pi_{1} \circ \psi_{2}$.
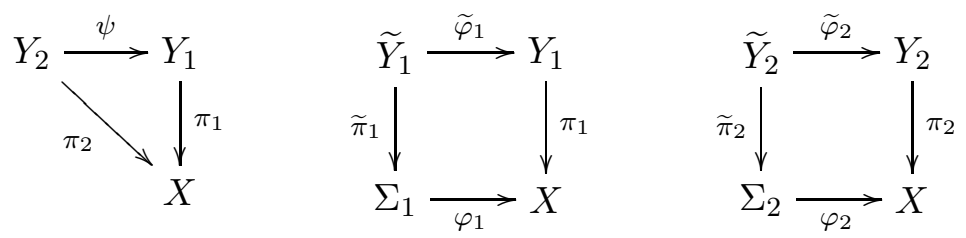

Lemma 3.7. Two generic triple covers $\pi_{1}$ and $\pi_{2}$ ramified over $C$ are equivalent if and only if the induced cyclic triple covers $\widetilde{\pi}_{1}$ and $\widetilde{\pi}_{2}$ are equivalent, and this equivalence induces also the equivalence of the canonical base changes $\varphi_{1}$ and $\varphi_{2}$.

Proof. Suppose $\pi_{1}$ is equivalent to $\pi_{2}$. Consider the following commutative diagram,

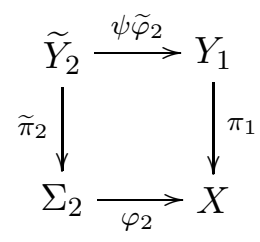

By the university property of fiber product, one can see that, in the diagram above, $\widetilde{Y}_{2}$ is also the normalization of $\Sigma_{2} \times_{X} Y_{1}$. Because $\widetilde{\pi}_{2}$ is Galois, by the uniqueness of $\varphi, \varphi_{2}$ is equivalent to $\varphi_{1}$. Note that $\pi_{2}$ is equivalent to $\pi_{1}$. Thus 
the fiber product of $\varphi_{2}$ with $\pi_{2}$ is isomorphic to the fiber product of $\varphi_{1}$ with $\pi_{1}$, it implies that $\widetilde{\pi}_{2}$ is equivalent to $\widetilde{\pi}_{1}$.

Conversely, suppose that $\widetilde{\pi}_{2}$ is equivalent to $\widetilde{\pi}_{1}$.
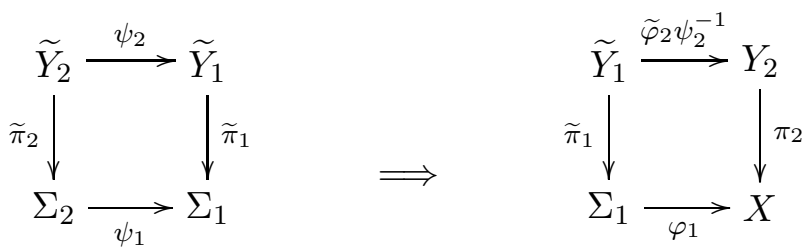

By the assumption, $\widetilde{\pi}_{1} \psi_{2}=\psi_{1} \widetilde{\pi}_{2}$ and $\varphi_{2}=\varphi_{1} \psi_{1}$. Similar to the above discussion, the commutative diagram on the right hand side is also a fiber product. So we can assume that $\Sigma_{2}=\Sigma_{1}, \varphi_{2}=\varphi_{1}, \widetilde{Y}_{2}=\widetilde{Y}_{1}, \widetilde{\pi}_{2}=\widetilde{\pi}_{1}, \psi_{1}$ and $\psi_{2}$ are identities.

Let $G \cong S_{3}$ be the Galois group of $\Pi_{1}: \widetilde{Y}_{1} \rightarrow X$, and let $Y_{1}=\widetilde{Y}_{1} / H_{1}$ and $Y_{2}=\widetilde{Y}_{1} / H_{2}$, where $H_{1}$ and $H_{2}$ are subgroups of $G$ of order 2 . Because $H_{2}$ is conjugate to $H_{1}$, there is an isomorphism $\psi: Y_{2} \rightarrow Y_{1}$ such that $\pi_{2}=\pi_{1} \circ \psi$. This completes the proof.

Therefore, in order to consider all of the equivalent generic triple covers ramified over a fixed cuspidal curve $C$, we can assume that they have the same canonical base changes $\varphi: \Sigma \rightarrow X$.

Corollary 3.8. Two generic triple covers $\pi_{1}$ and $\pi_{2}$ ramified over $C$ are equivalent if and only if $\widetilde{\pi}_{1}: \widetilde{Y}_{1} \rightarrow \Sigma$ and $\widetilde{\pi}_{2}: \widetilde{Y}_{2} \rightarrow \Sigma$ are equivalent.

Up to now, we have proved Theorem 0.5.

\section{Proof of Theorem 0.6}

We only need to prove that under the condition of Theorem 0.6 , if the cusps of $C$ are 3 -divisible of type $(\eta, \delta)$, then $3 \delta$ is determined uniquely by $C$. Equivalently, in the following relation,

$$
\sum_{i=1}^{n}\left(a_{i} E_{i}+a_{i}^{\prime} E_{i}^{\prime}\right) \equiv 3 \delta,
$$

$a_{i}$ and $a_{i}^{\prime}$ are determined uniquely by $C$. Then Theorem 0.6 follows from Lemma 3.6 and Corollary 3.8. 
In what follows, we assume that the above equation has two different sets of solutions $\left\{a_{i}, a_{i}^{\prime} \mid i=1, \cdots, n\right\}$ and $\left\{\bar{a}_{i}, \bar{a}_{i}^{\prime} \mid i=1, \cdots, n\right\}$.

Lemma 4.1. The $n$ cusps can be divided into two disjoint 3-divisible subsets.

Proof. We assume that the data corresponding to the two 3-divisible sets are

$$
\begin{aligned}
& \sum_{i=1}^{n}\left(E_{i}+2 E_{i}^{\prime}\right) \equiv 0 \quad(\bmod 3), \\
& \sum_{i=1}^{n_{1}}\left(E_{i}+2 E_{i}^{\prime}\right)-\sum_{i=n_{1}+1}^{n}\left(E_{i}+2 E_{i}^{\prime}\right) \equiv 0 \quad(\bmod 3) .
\end{aligned}
$$

Thus we get $\sum_{i=1}^{n_{1}}\left(E_{i}+2 E_{i}^{\prime}\right) \equiv 0(\bmod 3)$, and $\sum_{i=n_{1}+1}^{n}\left(E_{i}+2 E_{i}^{\prime}\right) \equiv 0(\bmod 3)$. This means that $\left\{p_{1}, \cdots, p_{n_{1}}\right\}$ and $\left\{p_{n_{1}+1}, \cdots, p_{n}\right\}$ are two 3 -divisible sets.

Note that the inequality in Theorem 0.4 holds for each of the above 3-divisible sets. Hence

$$
n \geq \frac{2 c_{1}^{2}}{3}+\frac{2 c_{1}^{2}}{3}=\frac{4 c_{1}^{2}}{3} .
$$

On the other hand, by Miyaoka-Yau inequality [15, 10, 22],

$$
n \leq \frac{1}{2}\left(9 \chi\left(\mathscr{O}_{\Sigma}\right)-K_{\Sigma}^{2}\right)
$$

By the standard formulas on double covers, we have

$$
\begin{aligned}
\chi\left(\mathscr{O}_{\Sigma}\right) & =2 \chi\left(\mathscr{O}_{X}\right)+\frac{1}{2}\left(c_{1}^{2}-c_{1} K_{X}\right), \\
K_{\Sigma}^{2} & =2\left(K_{X}-c_{1}\right)^{2} .
\end{aligned}
$$

Now by (4.1) and (4.2), we obtain

$$
c_{1}^{2}+3 c_{1} K_{X} \leq 12\left(9 \chi\left(\mathscr{O}_{X}\right)-K_{X}^{2}\right) .
$$

This is a contradiction. Theorem 0.6 is proved.

\section{Proof of Theorem 0.8}

Let $X$ be a smooth projective surface over $\mathbb{C}$, let $\mathscr{E}$ be a rank two vector bundle on $X$, and let $H$ be an ample divisor on $X$. We assume that $\mathscr{L}^{\vee} \cong \mathscr{O}_{X}(-L)$ is a maximal invertible subsheaf of $\mathscr{E}$ such that $\operatorname{deg}_{H} \mathscr{L}=c_{1}(\mathscr{L}) H$ is minimal. 
Denote by $Z_{0}$ the zero subscheme corresponding to a global section of $\mathscr{E} \otimes \mathscr{L}$, then the quotient $\mathscr{E} / \mathscr{L}^{\vee}$ is $\mathscr{I}_{Z_{0}}(-M), \operatorname{dim} Z_{0}=0$.

$$
0 \rightarrow \mathscr{L}^{\vee} \rightarrow \mathscr{E} \rightarrow \mathscr{I}_{Z_{0}}(-M) \rightarrow 0 .
$$

We have a surjective homomorphism $S^{3} \mathscr{E} \otimes(\operatorname{det} \mathscr{E})^{-2} \rightarrow \mathscr{I}_{Z_{1}}(2 L-M)$ given by $f \mapsto f_{0}$, where $Z_{1}$ is the base locus of $\left\{f_{0} \mid f\right\}$. Suppose $Z$ is the dimension one part, then $\mathscr{I}_{Z_{1}}=\mathscr{I}_{\Delta}(-Z)$ for some zero-dimensional subscheme $\Delta$. Let $\mathscr{F}$ be its kernel, then we have

$$
0 \rightarrow \mathscr{F} \rightarrow S^{3} \mathscr{E} \otimes(\operatorname{det} \mathscr{E})^{-2} \stackrel{I_{0}}{\rightarrow} \mathscr{I}_{Z_{1}}(2 L-M) \rightarrow 0,
$$

In order to relate $\mathscr{E}$ with a generic triple cover, we consider (5.2) for $\mathscr{E}_{m}=$ $\mathscr{E} \otimes \mathscr{O}_{X}(-m H)$ and $\mathscr{L}_{m}^{\vee}=\mathscr{L}^{\vee} \otimes \mathscr{O}_{X}(-m H) \subset \mathscr{E}_{m}$, we have

$$
0 \rightarrow \mathscr{F}_{m} \rightarrow S^{3} \mathscr{E}_{m} \otimes\left(\operatorname{det} \mathscr{E}_{m}\right)^{-2} \stackrel{I_{0}}{\rightarrow} \mathscr{I}_{Z_{1}}\left(2 L_{m}-M_{m}\right) \rightarrow 0,
$$

here $L_{m}=L+m H, M_{m}=M+m H, S^{3} \mathscr{E}_{m} \otimes\left(\operatorname{det} \mathscr{E}_{m}\right)^{-2}=S^{3} \mathscr{E} \otimes(\operatorname{det} \mathscr{E})^{-2} \otimes$ $\mathscr{O}_{X}(-m H)$ and $\mathscr{F}_{m}=\mathscr{F} \otimes \mathscr{O}_{X}(-m H)$. Namely, (5.3) is obtained from (5.2) by tensoring $\mathscr{O}_{X}(-m H)$.

Similarly, consider the homomorphism $J_{0}$, we get

$$
\begin{gathered}
0 \rightarrow \mathscr{F}^{\prime} \rightarrow S^{3} \mathscr{E} \otimes(\operatorname{det} \mathscr{E})^{-2} \stackrel{J_{0}}{\rightarrow} \mathscr{I}_{Z_{2}}(3 L) \rightarrow 0, \\
0 \rightarrow \mathscr{F}_{m}^{\prime} \rightarrow S^{3} \mathscr{E}_{m} \otimes\left(\operatorname{det} \mathscr{E}_{m}\right)^{-2} \stackrel{J_{0}}{\rightarrow} \mathscr{I}_{Z_{2}}\left(3 L_{m}\right) \rightarrow 0,
\end{gathered}
$$

where $\mathscr{F}_{m}^{\prime}=\mathscr{F}^{\prime} \otimes \mathscr{H}^{3 m}, \mathscr{I}_{Z_{2}}=\mathscr{I}_{\Delta^{\prime}}\left(-Z^{\prime}\right)$ and $\operatorname{dim} \Delta^{\prime}=0$.

Let $p: P(\mathscr{E}) \rightarrow X$ be the projective bundle of $\mathscr{E}, \mathscr{H}$ be the divisor on $P(\mathscr{E})$ associated to $\mathscr{O}_{P}(\mathscr{E})(1)$. We have

$$
H^{0}\left(X, \mathscr{E}_{m} \otimes\left(\operatorname{det} \mathscr{E}_{m}\right)^{-2}\right) \cong H^{0}\left(P(\mathscr{E}), \mathscr{O}_{P}(\mathscr{E})(\mathscr{D})\right),
$$

where

$$
\mathscr{D}=3 \mathscr{H}+p^{*}\left(m H-2 c_{1}(\mathscr{E})\right) .
$$

Note that when $m$ is big enough, $\mathscr{E}_{m} \vee$ is sufficiently ample. If we replace $\mathscr{E}$ by $\mathscr{E}_{m}$, then we can assume that the following statements hold true for $\mathscr{E}$.

(A) $(\operatorname{det} \mathscr{E})^{-1}$ is ample. $\mathscr{D}=3 \mathscr{H}+p^{*}\left(-2 c_{1}(\mathscr{E})\right)$ is very ample.

(B) $H^{1}(X, \mathscr{F})=0$, and $H^{1}\left(X, \mathscr{F}^{\prime}\right)=0$.

(C) $H^{1}\left(X, \mathscr{I}_{x} \otimes S^{3} \mathscr{E}(-2 \operatorname{det} \mathscr{E})\right)=0$ for any point $x \in X$. 
Cuspidal Curves and Vector Bundles on an Algebraic Surface, I 1805

(D) A generic element of $\left|\mathscr{I}_{Z_{1}}(2 L-M)\right|=\left|\mathscr{I}_{\Delta}(2 L-M-Z)\right|$ is an irreducible curve smooth on $X \backslash \Delta$.

(E) A generic element of $\left|\mathscr{I}_{Z_{2}}(3 L)\right|=\left|\mathscr{I}_{\Delta^{\prime}}\left(3 L-Z^{\prime}\right)\right|$ is an irreducible curve smooth on $X \backslash \Delta^{\prime}$.

Lemma 5.1. The generic member $Y$ of $|\mathscr{D}|$ is a smooth surface containing no fibers of $p$. So the induced morphism from $Y$ to $X$ is a triple cover $\pi: Y \rightarrow X$, and $\mathscr{E}=\mathscr{E}_{\pi}$ is the trace free subsheaf of $\pi_{*} \mathscr{O}_{Y}$.

Proof. We know that the restriction of $|\mathscr{D}|$ on the fiber $F_{x}=p^{*}(x) \cong \mathbb{P}^{1}$ of $p$ is $\mathscr{O}_{\mathbb{P}^{1}}(3)$.

$$
\begin{aligned}
H^{0}(P(\mathscr{E}), \mathscr{O}(\mathscr{D})) & \cong H^{0}\left(X, S^{3} \mathscr{E}(-2 \operatorname{det} \mathscr{E})\right) \\
H^{0}\left(P(\mathscr{E}), \mathscr{I}_{F_{x}}(\mathscr{D})\right) & \cong H^{0}\left(X, \mathscr{I}_{x} \otimes S^{3} \mathscr{E}(-2 \operatorname{det} \mathscr{E})\right)
\end{aligned}
$$

Note that $H^{1}\left(X, \mathscr{I}_{x} \otimes S^{3} \mathscr{E}(-2 \operatorname{det} \mathscr{E})\right)=0$. From the exact sequence

$$
\left.\left.0 \rightarrow \mathscr{I}_{x} \otimes S^{3} \mathscr{E}(-2 \operatorname{det} \mathscr{E})\right) \rightarrow S^{3} \mathscr{E}(-2 \operatorname{det} \mathscr{E})\right) \rightarrow \mathscr{O}_{x}^{\oplus 4} \rightarrow 0,
$$

we can see that

$$
h^{0}\left(P(\mathscr{E}), \mathscr{I}_{F_{x}}(\mathscr{D})\right)=h^{0}(P(\mathscr{E}), \mathscr{O}(\mathscr{D}))-4
$$

Now we let

$$
I=\left\{(S, x) \mid F_{x} \subset S\right\} \subset|\mathscr{D}| \times X,
$$

and let $\pi_{1}$ and $\pi_{2}$ be the first and second projection of $|\mathscr{D}| \times X$. By (5.6) we see that the dimension of the fiber of $\pi_{2}$ is $\operatorname{dim}|\mathscr{D}|-4$. We have

$$
\operatorname{dim} I \leq \operatorname{dim}|\mathscr{D}|-2 .
$$

Thus a generic surface in $|\mathscr{D}|$ does not contain any fiber of $F_{x}$. The trace free subsheaf of the induced triple cover is $\mathscr{E}$ (see Proposition 8.1 in [14], or the proof of Theorem 7.6 in [18]).

In what follows, we always assume that $f$ is generic in $P=P\left(H^{0}\left(X, S^{3} \mathscr{E} \otimes\right.\right.$ $\left.\operatorname{det} \mathscr{E}^{-2}\right)$ ), and $\pi(f): Y(f) \rightarrow X$ is the corresponding triple cover. By the assumptions, we have

1) $Y(f)$ is a smooth surface (by the previous lemma).

2) $\operatorname{div}\left(f_{0}\right)$ is an irreducible curve smooth on $X \backslash \Delta$.

3) $\operatorname{div}\left(\bar{f}_{0}\right)$ is an irreducible curve smooth on $X \backslash \Delta^{\prime}$. 
Lemma 5.2. The induced triple cover of the generic member of $P$ has no branch curve over which there is a total ramification.

Proof. Let

$$
\begin{aligned}
& \mathscr{B}=\{(f, x) \in P \times X \mid D(f)(x)=0\}, \\
& \mathscr{A}=\{(f, x) \in P \times X \mid H(f)(x)=0\} .
\end{aligned}
$$

For a fixed $x, D(f)(x)=0$ defines a hypersurface in $P$. The fiber $\mathscr{B}_{x}$ of $\mathscr{B}$ over $x$ has dimension $\operatorname{dim} P-1$, so $\operatorname{dim} \mathscr{B} \leq \operatorname{dim} \mathscr{B}_{x}+\operatorname{dim} X=\operatorname{dim} P+1$. Similarly, $H(f)(x)=0$ gives 2 independent conditions on $f$. We have $\operatorname{dim} \mathscr{A} \leq \operatorname{dim} P$.

It is easy to see that the projections $\mathscr{B} \rightarrow P$ and $\mathscr{A} \rightarrow P$ are surjective. Hence for a generic $f$ in $P$, the fiber $\mathscr{A}_{f}$ and $\mathscr{B}_{f}$ over $f$ satisfy

$$
\operatorname{dim} \mathscr{A}_{f}=0, \quad \operatorname{dim} \mathscr{B}_{f} \leq 1 .
$$

Note that $\mathscr{B}_{f}$ is the branch locus of $\pi(f)$, and $\mathscr{A}_{f}$ is the subset over which $\pi(f)$ is totally ramified. Then the lemma is proved.

From $H^{1}(X, \mathscr{F})=0$, we get a surjective homomorphism

$$
\begin{aligned}
& I_{0}: H^{0}\left(X, S^{3} \mathscr{E} \otimes(\operatorname{det} \mathscr{E})^{-2}\right) \rightarrow H^{0}\left(\mathscr{I}_{Z_{1}}(2 L-M)\right) \rightarrow 0 . \\
& f \quad \mapsto \quad f_{0}
\end{aligned}
$$

Note that $f_{0}=a_{2} b_{1} c_{0}$. By the assumption (D), the generic element of $\mid I_{Z_{1}}(2 L-$ $M) \mid$ is an irreducible curve and smooth on $X \backslash \Delta$. We take it as $A_{2}+B_{1}+C_{0}=$ $\operatorname{div}\left(f_{0}\right)$. From the above lemma, $A_{1}=A_{2}=0 . \bar{f}_{0}$ is the constant term in the cubic equation, $b_{1}^{2}$ is a factor of $\bar{f}_{0}$. Since $\operatorname{div}\left(\bar{f}_{0}\right)$ is irreducible, we have also that $B_{1}=0$. Thus $C_{0}=\operatorname{div}\left(f_{0}\right)$.

Lemma 5.3. For a generic $f$ in $P$, the branch locus $\mathscr{B}_{f}$ of $\pi(f): Y(f) \rightarrow X$ does not pass through any point in $\Delta$ or $\Delta^{\prime}$. Thus $B_{0}=\operatorname{div}\left(\bar{f}_{0}\right)$ and $C_{0}=\operatorname{div}\left(f_{0}\right)$ are smooth at any point on $\mathscr{B}_{f}$.

Proof. For any set $Z$ of finite points in $X$, the fibers $\mathscr{B}_{Z}$ of $\mathscr{B}$ over $Z$ satisfies

$$
\operatorname{dim} \mathscr{B}_{Z} \leq \operatorname{dim} P-1,
$$

which implies that the image of $\mathscr{B}_{Z}$ in $P$ is at most a divisor of $P$. So for a generic $f$ in $P, \mathscr{B}_{f} \cap \mathscr{B}_{Z}=\emptyset$, this means that $\pi(f)$ is unramified over $Z$. Let $Z=\Delta \cup \Delta^{\prime}$, we get the desired result. 
The branch locus $\mathscr{B}_{f}$ of $\pi(f)$ is defined by $D(f)=0$. Because $Y(f)$ is nonsingular, any singular point $p$ of the branch locus is a double point isomorphic to $x^{2}+y^{3 k_{0}}=0$, and $\pi(f)$ is totally ramified over $p$ ([18], Theorem 3.2). Therefore, the singular locus of the branch curve $\mathscr{B}_{f}$ is defined by $H(f)=0$, which is exactly $\mathscr{A}_{f}$. Because $\mathscr{A} \rightarrow P$ is a generic finite cover of degree $n$, for a generic $f, H(f)$ has $n$ different simple roots.

Lemma 5.4. Suppose $f$ is generic in $P$, and $H(f)$ has $n$ simple roots. Then the branch locus $\mathscr{B}_{f}$ has exactly $n$ ordinary cusps at the $n$ roots.

Proof. Suppose $p \in X$ is a simple root of $H(f)$, we have $h_{0}(p)=0$ and $D(f)(p)=$ 0 . From the syzygy

$$
4 h_{0}^{3}+\bar{f}_{0}^{2}=D(f) f_{0}^{2},
$$

we get $\bar{f}_{0}(p)=0$. Because $\operatorname{div}\left(\bar{f}_{0}\right)$ is nonsingular at $p$, we know that $f_{0}(p) \neq 0$. $D(f)$ is locally $x^{2}+y^{3 k_{0}}$, so $\bar{f}_{0}=0$ is the tangent of the double point. We assume that $\bar{f}_{0}=x$ is the local equation of the tangent. The local intersection number of $x$ with $h_{i}$ at $p$ is denoted by $k_{i}$. We need to prove that $k_{0}=1$.

From the assumption, the ideal $I=\left(h_{0}, h_{1}, h_{2}\right)$ of $\mathscr{O}_{X, p}$ is the maximal ideal $m_{p}=(x, y)$ of $\mathscr{O}_{X, p}$. It is easy to check that $\bar{f}_{0}=f_{0} h_{1}-2 f_{1} h_{0}$, we have

$(x, y)=I=\left(h_{0}, \bar{f}_{0}+2 f_{1} h_{0}, h_{2}\right)=\left(h_{0}, x, h_{2}\right)=\left(h_{0}(0, y), x, h_{2}(0, y)=\left(y^{k_{0}}, x, y^{k_{2}}\right)\right.$.

If $k_{0}>1$, then $k_{2}=1$. Since $h_{0}, h_{1}$ and $h_{2}=f_{1} f_{3}-f_{2}^{2}$ are the algebraic $2 \times 2$ minors of the matrix

$$
\left(\begin{array}{c}
f_{2}-f_{1} f_{0} \\
f_{3}-f_{2} f_{1}
\end{array}\right) .
$$

The rank of this matrix at $p$ is 1 . We see that if $f_{1}(p)=0$, then $f_{2}(p)=f_{3}(p)=0$, which implies that $p$ is a multiple point of $h_{2}$, hence $k_{2}>1$, a contradiction. Thus $f_{1}(p) \neq 0$. Now it is easy to see that $f_{2}(p)$ and $f_{3}(p)$ are nonzero. Since $f_{2} h_{0}-f_{1} h_{1}+f_{0} h_{2}=0$, we get $k_{1}=1$.

On the other hand, $D(f)=h_{1}^{2}-4 h_{0} h_{2}$, we see easily that the local intersection number of $D(f)$ with $x$ is $2 k_{1}=2$. We have known that $D(f)=x^{2}+y^{3 k_{0}}$, then the local intersection number should be $3 k_{0}>2$, a contradiction.

We have proved that $k_{0}=1$. Hence the singular points of the branch locus are ordinary cusps. 
Lemma 5.5. For a generic $f$ in $P$, the branch locus $D(f)=0$ is an irreducible curve.

Proof. We have known that all of the singular points of the branch curve $\mathscr{B}_{f}$ are ordinary cusps, so $\mathscr{B}_{f}$ is locally irreducible at any point. From $(\mathrm{A}), \mathscr{B}_{f} \equiv$ $-2 c_{1}(\mathscr{E})$ is ample. Now we claim that $\mathscr{B}_{f}$ is connected, by the local irreducibility, $\mathscr{B}_{f}$ must be irreducible.

Indeed, by the local irreducibility, if $\mathscr{B}_{f}=A+B$, then $A \cap B=\emptyset$. We have $A \mathscr{B}_{f}=A^{2}>0, B \mathscr{B}_{f}=B^{2}>0$. By Hodge index theorem, we have

$$
A^{2} \mathscr{B}_{f}^{2} \leq\left(A \mathscr{B}_{f}\right)^{2}, \quad B^{2} \mathscr{B}_{f}^{2} \leq\left(B \mathscr{B}_{f}\right)^{2},
$$

it implies

$$
\mathscr{B}_{f}^{2} \leq A \mathscr{B}_{f}, \quad \mathscr{B}_{f}^{2} \leq B \mathscr{B}_{f} .
$$

Thus

$$
2 \mathscr{B}_{f}^{2} \leq A \mathscr{B}_{f}+B \mathscr{B}_{f}=\mathscr{B}_{f}^{2},
$$

so $\mathscr{B}_{f}^{2} \leq 0$, which contradicts the ampleness of $\mathscr{B}_{f}$. This proves the lemma.

Thus we have proved Theorem 0.8.

\section{REFERENCES}

[1] Bogomolov, F.: Holomorphic tensors and vector bundles on projective varieties, Math. USSR, Izv. 13 (1979), 499-555

[2] Bronowski, J.: On Triple Planes, J. London Math. Soc., (I) 12 (1937), 212-216. (II) (1942) No.1, 24-31. (III) s1-17 (1942) no.2, 80-87

[3] Du Val, P.: On triple planes having branch curves of order not greater than twelve, J. London Math. Soc. s1-8 (1933) No.3, 199-206

[4] Du Val, P.: On triple planes whose branch curves are of order fourteen, Proc. London Math. Soc., s2-39 (1935) No.1, 68-81

[5] Chisini, O.: Sulla identità birationale delle funzioni algebriche di due variabili dotate di una medesima curva di diramazione. Rend. Istit. Lombardo 77 (1944), 339-356

[6] Chisini, O.: Courbes de diramation des plans multiples et tresses algébriques. Liege (Proc. 2nd Colloq. Algebraic Geom., Liege, 1952) 1952, Masson, Paris, 11-27

[7] Enriques, F.: Sulla costruzione delle funzioni algebriche di due variabili possedenti una data curve di diramazione. Annali di matematica pura ed applicata, 1 (1923) no.4, 185-198

[8] Friedman, R.: Algebraic surfaces and holomorphic vector bundles, Universitext, SpringerVerlag (1998)

[9] Fulton, W.: On the topology of algebraic varieties, in: Proc. Symp. Pure Math. 46, Part 1, Providence, RI: Amer. Math. Soc. (1987), 15-46 
[10] Hirzebruch, F.: Singularities of algebraic surfaces and characteristic numbers, in: The Lefschetz Centennial Conf. Part I (Mexico City 1984), Contemp. Math. 58 (1985), 141-155

[11] Kulikov, V.: On Chisini's conjecture, Izv. Math. 63 (1999) no.6, 1139-1170

[12] Kulikov, V.: On Chisini's conjecture, II, Izv. Math. 72 (2008) no.5, 901-913

[13] Libgober, A.: Fundamental groups of the complements to plane singular curves, in: Proc. Symp. Pure Math. 46, Part 2 Providence, RI: Amer. Math. Soc. (1987), 29-45

[14] Miranda, R.: Triple covers in algebraic geometry, Amer. J. Math. 107 (1985), 1123-1158

[15] Miyaoka, Y.: The maximal number of quotient singularities on surfaces with given numerical invariants, Math. Ann. 268 (1984), 159-171

[16] Nemirovskij, S. Yu.: Kulikov's theorem on the Chisini conjecture, Izv. Math. 65 (2001) no.1, 71-74

[17] Segre, B.: Sulla caratterizzazione delle curve di diramazione dei piani multipli generale, R. Accad. d'Ital. Cl. di Sci. Fis. Mat. et Nat., Memorie 1 (1930), 71-97

[18] Tan, S.-L.: Triple covers on smooth algebraic varieties, in: Geometry and Nonlinear Partial Differential Equations AMS/IP Studies in Advanced Mathematics 29 (2002), 143-164

[19] Tan, S.-L.: Binary forms in algebraic geometry, New Studies in Advanced Mathematics, 4 (2004), 187-198

[20] Tan, S.-L., Teicher, M.: On the spaces of branch curves of triple planes, preprint

[21] Tan, S.-L., Zhang, D.-Q.: The determination of integral closure and geometric applications, Adv. in Math., 185 (2004), 215-245

[22] Yau, S.-T.: On the Ricci curvature of a compact Kähler manifold and the complex MongeAmpère equation. I, Comm. on Pure and Applied Math. 31 (1978) no.3, 339-411

[23] Zariski, O.: On the problem of the existence of algebraic functions, Amer. J. Math. 51 (1929), 305-328

Sheng-Li Tan

Department of Mathematics,

East China Normal University,

Dongchuan RD 500,

Shanghai 200241, P. R. of China

E-mail: sltan@math.ecnu.edu.cn 